\title{
Molecular Diagnosis of Koala Retrovirus (KoRV) in South Australian Koalas (Phascolarctos cinereus)
}

\author{
Tamsyn Stephenson ${ }^{1, *(\mathbb{D})}$, Natasha Speight ${ }^{1} \mathbb{D}$, Wai Yee Low ${ }^{2} \mathbb{D}$, Lucy Woolford ${ }^{1,3}$, Rick Tearle $^{2}$ \\ and Farhid Hemmatzadeh ${ }^{1}$ (D)
}

1 School of Animal and Veterinary Sciences, University of Adelaide, Roseworthy 5371, Australia; natasha.speight@adelaide.edu.au (N.S.); lucy.woolford@adelaide.edu.au (L.W.); farhid.hemmatzadeh@adelaide.edu.au (F.H.)

2 The Davies Livestock Research Centre, School of Animal and Veterinary Sciences, University of Adelaide, Roseworthy 5371, Australia; wai.low@adelaide.edu.au (W.Y.L.); rick.tearle@adelaide.edu.au (R.T.)

3 Veterinary Diagnostics Laboratory, School of Animal and Veterinary Sciences, University of Adelaide, Roseworthy 5371, Australia

* Correspondence: tamsyn.stephenson@adelaide.edu.au

Citation: Stephenson, T.; Speight, N.; Low, W.Y.; Woolford, L.; Tearle, R.; Hemmatzadeh, F. Molecular

Diagnosis of Koala Retrovirus (KoRV) in South Australian Koalas (Phascolarctos cinereus). Animals 2021, 11, 1477. https://doi.org/10.3390/ ani11051477

Academic Editor: Seiya Yamayoshi

Received: 8 April 2021

Accepted: 16 May 2021

Published: 20 May 2021

Publisher's Note: MDPI stays neutral with regard to jurisdictional claims in published maps and institutional affiliations.

Copyright: (c) 2021 by the authors. Licensee MDPI, Basel, Switzerland. This article is an open access article distributed under the terms and conditions of the Creative Commons Attribution (CC BY) license (https:// creativecommons.org/licenses/by/ $4.0 /)$.
Simple Summary: Koala retrovirus (KoRV) is a significant threat to koalas across Australia. Koalas in northern koala populations (from New South Wales and Queensland) have KoRV inserted into their DNA and inherited to their offspring. Southern koala populations (from Victoria and South Australia) have KoRV infection spread through close contact of koalas. As such, there are koalas within South Australia that are not infected with KoRV. Accurate diagnosis of the infection of each koala is therefore fundamental for disease studies. Previous studies have shown differences in prevalence of different KoRV genes in the Mount Lofty Ranges Koala population; therefore, clarification is necessary. This study uses a large cohort $(n=216)$ and defines the diagnostic regions of the KoRV genome within the South Australian population. Using multiple molecular techniques, it demonstrates strong evidence for two clear groupings of koalas: KoRV positive and KoRV negative. Within this study, a population of $41 \%$ were shown to be KoRV positive and $57 \%$ were KoRV negative, with $2 \%$ inconclusive. This differentiation is of great importance when examining the clinical importance of KoRV infection within southern koalas.

Abstract: Koala retrovirus, a recent discovery in Australian koalas, is endogenised in 100\% of northern koalas but has lower prevalence in southern populations, with lower proviral and viral loads, and an undetermined level of endogenisation. KoRV has been associated with lymphoid neoplasia, e.g., lymphoma. Recent studies have revealed high complexity in southern koala retroviral infections, with a need to clarify what constitutes positive and negative cases. This study aimed to define KoRV infection status in Mount Lofty Ranges koalas in South Australia using RNA-seq and proviral analysis $(n=216)$. The basis for positivity of KoRV was deemed the presence of central regions of the KoRV genome ( $g a g$ 2, pol, env 1, and env 2) and based on this, 41\% (89/216) koalas were positive, 57\% (124/216) negative, and $2 \%$ inconclusive. These genes showed higher expression in lymph node tissue from KoRV positive koalas with lymphoma compared with other KoRV positive koalas, which showed lower, fragmented expression. Terminal regions (LTRs, partial gag, and partial env) were present in SA koalas regardless of KoRV status, with almost all (99.5\%, 215/216) koalas positive for gag 1 by proviral PCR. Further investigation is needed to understand the differences in KoRV infection in southern koala populations.

Keywords: koala retrovirus; KoRV; koala; Phascolarctos cinereus; diagnosis; PCR; qPCR; South Australia 


\section{Introduction}

The genome of koala retrovirus (KoRV) was sequenced in 2000 [1] and was shown to be a type-C gammaretrovirus. It is simple in structure, approximately $8.4 \mathrm{~kb}$ long, and contains three genes: group-specific antigen gene ( $\mathrm{gag}$ ), protease-polymerase gene ( $p r o-p o l$ or $p o l)$, and the envelope gene (env), flanked by long terminal repeat (LTR) regions [1]. The gag gene encodes matrix, capsid, and nucleocapsid proteins and is necessary for the structure [2] of KoRV and its ability to create budding virions [3]; the pol gene codes for reverse transcriptase, integrase and protease enzymes; and the env gene encodes surface and transmembrane proteins from which cell tropism and receptor affinity arise [2]. KoRV variants, denoted A-I, have been classified into three major clades, based on the hypervariable region of the env gene; KoRV-A, KoRV-B, and KoRV C-I [4-11]. KoRV-A is known to enter the cell via the Pit-1 (SLC20A1) receptor [12], KoRV-B via the THTR1 receptor [3,5], and the other KoRV subtypes receptors through an unknown mechanism. KoRV has two fundamental methods of transmission: endogenously through the germ line from parent to offspring [11]; and exogenously, via horizontal (close contact) or vertical (mother to joey in the pouch) transmission [5,9]. Northern mainland koala populations (from Queensland and New South Wales) have all been found to be positive for endogenous KoRV-A provirus, with exogenous variants KoRV-B through to KoRV-I also present in some koalas [8]. In contrast, southern koala populations (from Victoria and South Australia) have a lower prevalence of KoRV, primarily KoRV-A, and have lower proviral and viral loads. This has led to the theory that these populations are currently undergoing the process of KoRV endogenisation, with predominantly KoRV-A spreading exogenously through this population [8,13-18], but this is yet to be confirmed.

Studies of KoRV in South Australian (SA) koalas have been based on detection of KoRV provirus and virus using a PCR amplification of part of the pol gene, a highly conserved region of the KoRV genome. Based on this, large numbers of KoRV-free koalas have been found in the two main populations of SA koalas in Kangaroo Island (KI) and the Mount Lofty Ranges (MLR). However more recent studies utilising PCR targeting of gag and env genes, as well as expression studies of the entire KoRV genome, have identified a more complex KoRV profile in MLR koalas [16,17], with 38-99\% positive for nine different proviral targets, and $3-51 \%$ positive for viral RNA targets [17]. It has also been shown that MLR KoRV pol-negative koalas produced transcripts that aligned to the KoRV genome at positions 1-1389 (5' LTR and partial gag gene) and 7124-8431 (partial env gene and 3'LTR), with no transcription of other KoRV genes [19]. Additionally, KoRV pol-positive koalas had low transcription of genes between genome positions 1389 and 7124, representing the $5^{\prime}$ end of the gag gene, the pol gene and the $3^{\prime}$ end of the env gene, unlike Queensland koalas [19]. It is currently postulated that truncated and potentially defective virus inhibits viral replication in these koalas [16,19]. Another recent study has found that terminal sequences from KoRV have, in some cases, recombined with earlier or ancient retroviral elements, such as Phascolarctid endogenous retroelement (PhER) [20,21]. PhER has been shown to still be active and expressing at least parts of genes [20,21], although the clinical significance of this is unknown. This recombination of KoRV with PhER, has been designated as recKoRV and has one prominent subpopulation designated recKoRV-1. RecKoRV-1 has been shown to be present in the MLR population, but not the KI population and notably, within the MLR population recKoRV-1 was shown to be present in KoRV pol negative koalas [20].

In northern koalas, disease states that have been associated with KoRV infection include lymphoid neoplasia (lymphoma and leukaemia) and chlamydiosis [15,22-24]. Lymphoid neoplasia and increased severity of chlamydiosis have been found to be associated with high proviral and viral loads in all regions $[15,17,23]$ and with exogenous KoRV-B infection in northern koalas $[4,5,24]$. In the MLR population lymphoma has been found in koalas with KoRV viral and proviral loads comparable to those of northern koalas with neoplastic disease [17]. No significant association has been identified between KoRV-A status and Chlamydia pecorum status in MLR koalas [13,22], despite the exogenous transmission in this population and the suggested increased pathogenicity of exogenous KoRV infections. 
A study in KoRV positive koalas did show a positive correlation between KoRV viral load and severity of chlamydiosis [25], indicative of potentially increased pathogenicity with higher viral loads.

KoRV infection in SA koalas is clearly complex, and hence it is important to clarify which KoRV genes should be used to identify an individual as KoRV positive or negative, in order to understand infection prevalence and interpret disease in SA koalas. The aims of this study were to describe the integration and expression of KoRV genes in MLR koalas, so as to recommend guidelines for classification of individuals as positive or negative for infectious, full length KoRV in SA.

\section{Materials and Methods}

\subsection{Samples}

Koalas for this study $(\mathrm{n}=216)$ were sourced from the Mount Lofty Ranges population in South Australia following euthanasia by veterinarians on welfare grounds. All koalas received a full necropsy at the Veterinary Diagnostic Laboratory, Roseworthy campus, University of Adelaide. Blood samples taken prior to euthanasia were stored in EDTA tubes (Vacuette ${ }^{\circledR}$ Tube, Greiner Bio-One GmBH, Kremsmünster, Austria), or spleen samples were taken postmortem, and stored at $-20{ }^{\circ} \mathrm{C}$ prior to DNA analysis. In koalas that were available for immediate postmortem examination $(<1 \mathrm{~h})$, small $0.5 \mathrm{~cm}^{2}$ pieces of lymph nodes were placed into RNA Later ${ }^{\circledR}$ (Sigma Aldrich, Saint Louis, MO, USA), refrigerated for $24 \mathrm{~h}$ and stored at $-80^{\circ} \mathrm{C}$ prior to RNA analysis. All lymph nodes were examined histologically to determine the presence or absence of lymphoma, and if present, assigned to the lymphoma group $(\mathrm{n}=6)$.

\subsection{DNA Analysis for KoRV Provirus: DNA Extraction}

DNA was extracted from whole blood or spleen for KoRV proviral analysis, using the QIAMP DNeasy Minikit (Qiagen, Hilgen, Germany) as per manufacturer's instructions. All extracted DNA was quantified using a NanoDrop One spectrophotometer (Thermo Fisher Scientific Inc, Wilmington, DE, USA) and stored at $-20^{\circ} \mathrm{C}$.

\subsection{DNA Analysis for KoRV Provirus: DNA Proviral PCR and $q P C R$}

KoRV proviral gene targets gag 1, gag 2, env 1, and env 2 (Figure 1) were assessed by conventional PCR using DNA extracted from either whole blood or spleen. KoRV gag 1 and 2 were optimised as a multiplex reaction and env 1 and 2 reactions were run individually. The KoRV gag (1 and 2) $25 \mu \mathrm{L}$ PCR reactions consisted of $5 \mu \mathrm{L}$ AllTaq Mastermix (Qiagen, Hilden, Germany), $0.25 \mu \mathrm{M}$ of each gag 1 primer, $0.75 \mu \mathrm{M}$ of each gag 2 primer (Table 1 ), $3 \mu \mathrm{L}$ of $1 / 10$ dilution DNA template, and $9 \mu \mathrm{L}$ of PCR-grade water. The individual KoRV env $20 \mu \mathrm{L}$ PCR reactions consisted of $4 \mu \mathrm{L}$ AllTaq Mastermix (Qiagen, Hilden, Germany), $0.5 \mu \mathrm{M}$ of each primer (Table 1), $3 \mu \mathrm{L}$ of $1 / 10$ dilution DNA template and $10 \mu \mathrm{L}$ of PCR-grade water. The temperature and timing of the PCR reactions for both assays were initial activation and denaturation of $95^{\circ} \mathrm{C}$ for $3 \mathrm{~min} ; 40$ cycles of denaturation at $95^{\circ} \mathrm{C}$ for $5 \mathrm{~s}$; annealing at $60^{\circ} \mathrm{C}$ (KoRV env) or $62{ }^{\circ} \mathrm{C}$ (KoRV gag) for $15 \mathrm{~s}$; and extension at $72{ }^{\circ} \mathrm{C}$ for $10 \mathrm{~s}$. This was followed by a final extension step of $72{ }^{\circ} \mathrm{C}$ for $10 \mathrm{~s}$. Gel electrophoresis using $1.0 \%$ agarose gel was carried out and PCR products visualised under UV light. Samples were loaded in combination with gel red, producing bands at the appropriate product size (Table 1).

KoRV proviral load was measured in DNA extracted from either whole blood or spleen by KoRV pol gene qPCR (Table 1, Figure 1). Koala $\beta$-actin was used as the reference gene. qPCR reactions of $5 \mu \mathrm{L}$ were set up in triplicate using an Eppendorf epMotion $5075 \mathrm{LH}$ model (Eppendorf, Hamburg, Germany). Each reaction consisted of $2.5 \mu \mathrm{L}$ of Power SYBR green Master Mix (Applied Biosystems, Foster City, CA, USA), $300 \mathrm{nM}$ of each primer and $1.5 \mu \mathrm{L}$ of $1 / 10$ or $1 / 100$ dilution of DNA template. qPCRs were run on a 7900 HT Sequence Detection System (Applied Biosystems, Singapore). Reaction conditions were initial denaturation $95^{\circ} \mathrm{C}$ for $10 \mathrm{~min}$; 40 cycles of $95^{\circ} \mathrm{C}$ for $15 \mathrm{~s} ; 60^{\circ} \mathrm{C}$ for $60 \mathrm{~s}$. Melt curve analysis was carried out at $95{ }^{\circ} \mathrm{C}$ for $15 \mathrm{~s}, 60{ }^{\circ} \mathrm{C}$ for $15 \mathrm{~s}$ and $95{ }^{\circ} \mathrm{C}$ for $15 \mathrm{~s}$. Copy number was calculated from a 
standard curve derived from purified PCR products for both KoRV pol and $\beta$-actin from koala lymphoma tissue. KoRV proviral load reported as copies $/ 10^{3} \beta$-actin copies.

\begin{tabular}{|c|c|c|c|c|c|c|c|}
\hline & $\underset{\mathrm{D}}{\operatorname{gag} 1}$ & $\stackrel{g a g}{ } 2$ & pol & & $\stackrel{e n v 1}{\square}$ & 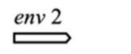 & \\
\hline 5'LTR & & GAG & & POL & 60245906 & ENV & 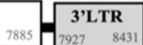 \\
\hline
\end{tabular}

Figure 1. Representation of the KoRV genome (AF151794); showing genes, coordinates and primer locations with block arrows indicating amplicon length.

Table 1. PCR targets and primers.

\begin{tabular}{|c|c|c|c|}
\hline Target & Primers & Product Size & Reference \\
\hline Koala $ß$-actin & $\begin{array}{l}\text { Fwd 5'-GAGACCTTCAACACCCCAGC-3' } \\
\text { Rev 5'-GTGGGTCACACCATCACCAG-3' }\end{array}$ & $111 \mathrm{bp}$ & Shojima et al. 2013 [4] \\
\hline KoRV gag 1 & $\begin{array}{l}\text { Fwd 5' -CGGACCAGGTTCCCTACATC-3' } \\
\text { Rev 5'- TCGCCCGTTATCTTGACCAG-3' }\end{array}$ & $110 \mathrm{bp}$ & This study \\
\hline KoRV gag 2 & $\begin{array}{l}\text { Fwd 5'-TTGGCCTTTCTCCTCAGCAG-3' } \\
\text { Rev 5'-CCGTGTTGTGATCCCACTGA-3' }\end{array}$ & $290 \mathrm{bp}$ & This study \\
\hline KoRV pol & $\begin{array}{l}\text { Fwd 5'-TTGGAGGAGGAATACCGATTACAC-3' } \\
\text { Rev 5'-GCCAGTCCCATACCTGCCTT-3 }{ }^{\prime}\end{array}$ & $111 \mathrm{bp}$ & Tarlinton et al. 2005 [23] \\
\hline KoRV-A env 1 & $\begin{array}{l}\text { Fwd 5'-TCCTGGGAACTGGAAAAGAC-3' } \\
\text { Rev 5'-GGGTTCCCCAAGTGATCTG-3' }\end{array}$ & $321 \mathrm{bp}$ & Waugh et al. 2017 [24] \\
\hline KoRV env 2 & $\begin{array}{l}\text { Fwd 5'-GCCCTCGGCCCTCCTTATTA-3' } \\
\text { Rev 5'-GGCAATCTGGAGGCTAGTCAA-3 }{ }^{\prime}\end{array}$ & $522 \mathrm{bp}$ & Sarker et al. 2020 [17] \\
\hline
\end{tabular}

\subsection{RNA Extraction and Sequencing}

RNA was extracted to analyse KoRV gene expression by RNAseq and qPCR. Lymph nodes (lymphomic and non-lymphomic) from eight SA koalas, two KoRV proviral positive with lymphoma, three KoRV proviral positive, and three KoRV proviral negative (except gag 1 ), were used. Tissues were thawed on ice, disrupted with zirconium oxide beads $(0.5 \mathrm{~mm}$, RNA-free, BioTools, Loganholme, Australia) in a Bullet Blender and Tissue Homogeniser (Next Advance, Troy, NY 12180, USA)), and then cooled on ice. The mirVana ${ }^{\mathrm{TM}}$ kit (Thermo Fisher Scientific) was used to extract total mRNA as per the manufacturer's protocol. RNA integrity (RIN) was checked using a 2100 BioAnalyser (Agilent Technologies). cDNA libraries were made and sequenced using an Illumina NovaSeq sequencing platform that generated $2 \times 150$ bp reads (AGRF, Melbourne, Australia).

\subsection{KoRV Gene Expression from RNA-seq Analysis}

The RNA-seq reads were cleaned using Trim_Galore (v0.4.2) [26] and AdapterRemoval (v2.2.1) [27] to remove adapters and bases with a Phred score less than 10. Fastqc (v0.11.4) [28] was used to check the quality of the cleaned reads. The reads were aligned to the koala reference genome (GCA_002099425.1_phaCin_unsw_v4.1) using HISAT2 (v2.1.0) [29].

\subsection{BLAST Analysis}

To demonstrate the presence of similar KoRV termini regions to those found in KoRV negative koalas, NCBI standard nucleotide Basic Local Alignment Search Tool (BLASTn) [30] was used to align the KoRV-A (AF151794.2) reference genome against the koala reference genome (phaCin_unsw_v4.1 reference Annotation Release 100; GCF_002099425.1). Further BLASTn analysis was carried out on alignments to the koala genome that only contained the terminal regions of KoRV homology. Additionally, the central "gap" sequences were 
recovered from between these terminal alignments from the reference genome and BLASTn was then used to align these gap sequences to the nucleotide NCBI database [31]. BLASTn was also used to align the Phascolarctos endogenous retroelement (PhER), using coordinates reported in Lober et al. 2018 [20], against the koala reference genome. PhER and KoRV alignment results were then compared to find any crossover regions.

\subsection{Viral Two Step RT-qPCR Analysis}

To quantify the difference in KoRV viral gene expression between the three groups, total mRNA was extracted from 15 koala's lymph nodes, five KoRV proviral positive with lymphoma (Lymphoma), five KoRV proviral positive (Positive), and five KoRV proviral negative (except gag 1) (Negative). cDNA was reverse transcribed from 250-2500 ng of total RNA using SuperScript IV First Strand Synthesis system (Invitrogen, Thermofisher Scientific) and oligoDT primers as per manufacturer's instructions. cDNA was diluted to a working solution based on starting RNA (equivalent to $1.25 \mathrm{ng} / \mu \mathrm{L}$ RNA). qPCR reactions were run, targeting four KoRV gene regions; gag 1, gag 2, pol, and env 1, with primers described in Table 1 and targets shown on the KoRV genome in Figure 1. Koala $\gamma$-actin was used as the reference gene; forward primer $5^{\prime}$-TGCGCAGCTTCAGATTAAACAA$3^{\prime}$, reverse primer $5^{\prime}$-GGCCTCATCACCAACATAACTG-3' ${ }^{\prime}$, due to increased stability in comparison to koala $B$-actin across RNA-seq data for the cohorts. Coefficients of variance $(\mathrm{CoV})$ [32] were calculated from gene expression normalised counts (cpm) across all samples with $\gamma$-actin $\mathrm{CoV}$ calculated as 0.04 (position 289/14435) in comparison to ß-actin CoV 0.06 (position 906/14435). qPCR was optimised for $\gamma$-actin and conditions for the qPCR are as follows; $5 \mu \mathrm{L}$ reactions were set up in triplicate using an Eppendorf epMotion $5075 \mathrm{LH}$ model (Eppendorf, Hamburg, Germany). Each reaction consisted of $2.5 \mu \mathrm{L}$ Power SYBR green Master Mix (Applied Biosystems, Foster City, CA, USA). $300 \mathrm{nM}$ of each primer (Table 1) and $1.5 \mu \mathrm{L}$ of cDNA template. qPCRs were run on a 7900 HT Sequence Detection System (Applied Biosystems, Singapore). Reactions conditions were initial denaturation $95^{\circ} \mathrm{C}$ for $10 \mathrm{~min}$, followed by 40 cycles of $95^{\circ} \mathrm{C}$ for $15 \mathrm{~s}, 60^{\circ} \mathrm{C}$ for $60 \mathrm{~s}$. Melt curve analysis was carried out with conditions of $95^{\circ} \mathrm{C}$ for $15 \mathrm{~s}, 60^{\circ} \mathrm{C}$ for $15 \mathrm{~s}$, and $95^{\circ} \mathrm{C}$ for $15 \mathrm{~s}$.

\subsection{Relative qPCR Statistical Analysis}

Comparative CT $\left(2^{(-\Delta \Delta C t)}\right)$ was calculated for each of the four gene targets studied. The Mann-Whitney U Test was used to determine significant differences between groups. Both calculations were carried out in Microsoft Excel (Version 16.42, 2020).

\section{Results}

\subsection{Proviral DNA Analyses}

The presence/absence of three KoRV genes was determined by PCR using five targets, two in the gag gene, one in the pol gene and two in the env gene, the targets called gag 1 , gag 2, pol, env 1, and env 2, respectively. The majority of koalas (212/216) could be placed into two categories: positive for all gene targets or negative for all gene targets except gag 1 (Table 2). The remaining koalas $(4 / 216)$ showed variation in their gene target results with one koala negative for all targets (Table 2).

Table 2. Proviral PCR results.

\begin{tabular}{|c|c|c|c|c|c|c|c|}
\hline \multicolumn{5}{|c|}{ KoRV Gene Target } & \multirow{2}{*}{$\begin{array}{l}\text { Count } \\
(n=216)\end{array}$} & \multirow{2}{*}{$\%$} & \multirow{2}{*}{$\begin{array}{l}\text { Median (Range) of Proviral Load KoRV Copies/ } \\
10^{3} \text { B-Actin Copies * }\end{array}$} \\
\hline $\operatorname{gag} 1$ & gag 2 & pol & env 1 & env 2 & & & \\
\hline+ & + & + & + & + & 89 & 41.2 & $41(0.2-270)$ \\
\hline+ & - & - & - & - & 123 & 56.9 & 0 \\
\hline+ & - & - & - & + & 2 & 0.9 & 0 \\
\hline+ & - & - & + & - & 1 & 0.5 & 0 \\
\hline- & - & - & - & - & 1 & 0.5 & 0 \\
\hline
\end{tabular}




\subsection{KoRV Gene Expression from RNA-seq Analysis}

Figure 2 shows the KoRV expression profiles for the lymph nodes of eight koalas as determined by mapping the RNA-seq reads to a KoRV insertion found in the reference koala genome (scaffold 27:2807277-2815707 of GCA_002099425.1_phaCin_unsw_v4.1). Every RNAseq sample, regardless of lymphoma and KoRV PCR status, showed substantial coverage across the LTRs and nearby gene regions (herein called the terminal regions).

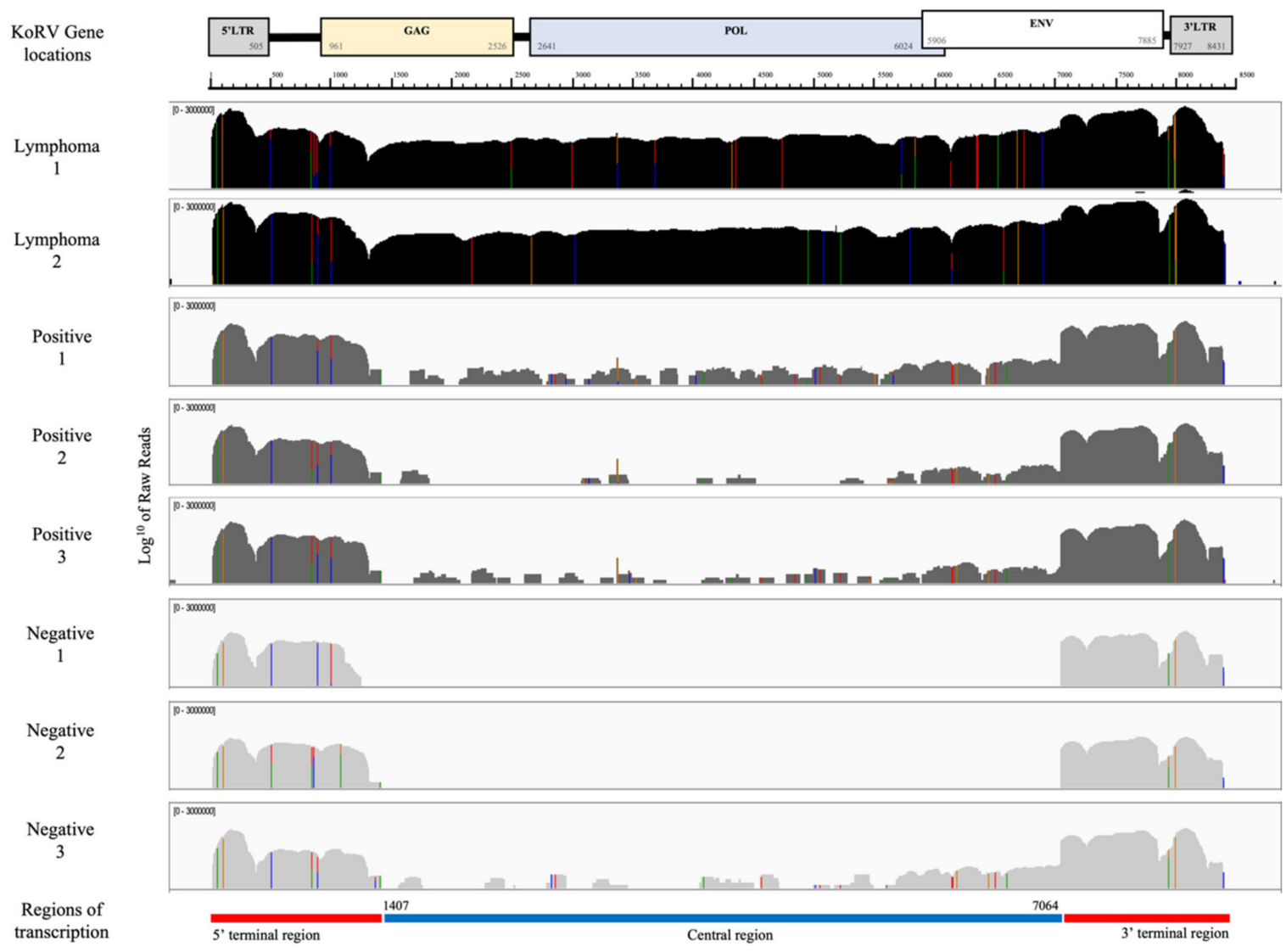

Figure 2. Transcription expression profile (mapped cleaned reads) for two koalas with lymphoma, three KoRV proviral positive and three KoRV proviral negative (except gag 1) koalas, demonstrating alignment to the KoRV genome, location of primers for proviral PCRs and transcription regions. All expression is a $\log _{10}$ scale of read counts, black represents KoRV proviral positive koalas diagnosed with lymphoma, dark grey represents KoRV proviral positive koalas and light grey represents KoRV proviral negative (except gag 1) koalas. All mapped to scaffold 27; 2807277-2815707 (GCA 002099425.1 phaCin_unsw_v4.1), KoRV-A accession AF151794.

Two positive koalas with lymphoma (Lymphoma 1 and 2) also showed substantial, comparable coverage across the region between them (herein called the central region), supporting previous findings of higher KoRV transcription and viral loads in lymphoid neoplasia [23]. Three koalas negative for lymphoma but PCR positive for all five proviral gene targets (Positive 1,2 and 3) only showed only low, fragmented coverage across the central region. Low loads of KoRV are likely due to only exogenous infections assumed in South Australia [10], and possibly subclinical retroviral status. Of three koalas negative for all proviral PCR targets except gag 1 , the koalas Negative 1 and 2 showed no coverage across the central region, but Negative 3 showed low, fragmented coverage. No transcription and no provirus found in the central region would deem these koalas unable to produce virions and support their KoRV negative infections status. Negative 3 koala could be an acute or early-stage infection, potentially due to the target tissue of lymph nodes from which the RNA was extracted for RNAseq, whereas proviral analysis was carried out on blood or 
spleen tissue; alternatively, this may reflect a KoRV positive koala whose proviral load is too low for PCR positivity.

\subsection{Terminal Regions of Homology to KoRV}

Alignment of KoRV-A (AF151794.2) against the reference koala genome (phaCin_unsw _v4.1 reference Annotation Release 100; GCF_002099425.1) using BLASTn showed two types of alignments, a smaller subset of those found in Hobbs et al. [33]: nine alignments were to the whole or most of KoRV, but eight only aligned to the terminal regions of KoRV. The terminal alignments were found in scaffolds, 137, 005, 288, 113, 073, 357, 253, and 101 (coordinates in Supplementary Material: Table S2). The terminal region alignment lengths varied from $1155-2663 \mathrm{bp}$ in the $5^{\prime}$ region and 812-2919 bp in the $3^{\prime}$ region and are quite similar to the consistently expressed terminal regions in Figure 2. The central regions within these scaffolds, from the reference genome, were then aligned using BLASTn against the nucleotide database. Alignments were found to uncharacterised loci in the koala, wombat (Vombatus ursinus) and gray short-tailed opossum (Monodelphis domestica) genomes. However, alignment of PhER (phaCin_unsw_v4.1.fa.scaf00062: 10912078-10920108, as per Lober et al. 2018 [20]) using BLASTn against the koala reference genome revealed alignment to the same scaffolds and positions of the central gap region of KoRV (coordinates in Supplementary Material: Table S3). Overall, the BLASTn data shows that similar terminal regions are present in the northern koala reference genome and the "gap" region, in the identified scaffolds, aligns to PhER (Figure 3), reiterating findings in Lober et al. [20] and Hobbs et al. [33]. The significant similarity of terminal fragments in the reference genome and within the KoRV negative koalas in SA is suggestive of the presence of a recombinant KoRV (recKoRV) in these koalas.

\begin{tabular}{|c|c|c|c|}
\hline \multirow{2}{*}{ Scaffold: 137 (7148 bp) } & $4,938,042$ & & $4,945,1$ \\
\hline & $99.88 \%$ & \multirow[b]{2}{*}{$96.16 \%$} & $99.13 \%$ \\
\hline \multirow{2}{*}{ Scaffold: 005 (6850 bp) } & $26,672,908$ & & $26,679,758$ \\
\hline & $99.88 \%$ & \multirow[b]{2}{*}{$96.06 \%$} & $99.07 \%$ \\
\hline \multirow{2}{*}{ Scaffold: 288 (6835 bp) } & 726,495 & & 733,330 \\
\hline & $99.88 \%$ & \multirow{2}{*}{$96.15 \%$} & $99.24 \%$ \\
\hline \multirow{2}{*}{ Scaffold: 113 (6836 bp) } & $3,968,195$ & & $3,975,031$ \\
\hline & $99.02 \%$ & \multirow{2}{*}{$96.32 \%$} & $98.99 \%$ \\
\hline \multirow{2}{*}{ Scaffold: 073 (6831 bp) } & $4,193,643$ & & $4,200,474$ \\
\hline & $99.88 \%$ & \multirow[b]{2}{*}{$96.34 \%$} & $99.15 \%$ \\
\hline \multirow{2}{*}{ Scaffold: 357 (6834 bp) } & 128,022 & & 134,856 \\
\hline & $99.88 \%$ & \multirow{2}{*}{$96.28 \%$} & $99.07 \%$ \\
\hline \multirow{2}{*}{ Scaffold: 253 (6833 bp) } & $2,925,255$ & & $2,932,088$ \\
\hline & $99.88 \%$ & & $9988 \%$ \\
\hline
\end{tabular}

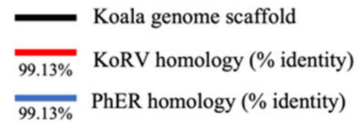

Figure 3. BLASTn analysis results showing crossover of the central gap regions from alignment of KoRV termini regions (red) and PhER (blue) to the koala reference genome (black). KoRV is represented by the reference sequence for KoRV-A: AF151794, PhER: phaCin_unsw_v4.1. fa.scaf00062:10912078-10920108, as per Lober et al. 2018 [20] and the reference koala genome: phaCin_unsw_v4.1 reference Annotation Release 100; GCF_002099425.1.

\subsection{KoRV Gene Expression from Viral Two Step RT-qPCR Analysis}

qPCR for KoRV gene targets gag 1, gag 2, pol, and env 1 were carried out on RNA from fifteen koala lymph nodes, five KoRV positive with lymphoma (three of which were from a previous study cohort [25]), five KoRV positive, and five KoRV negative koalas. Seven of the eight koalas that were previously examined by RNA-seq transcriptome analysis were examined by viral RT-qPCR (all except Negative 3, as not enough material remained). Because gag 1 was positive in almost every test, regardless of the sample lymphoma and KoRV status, it was excluded from further analysis. Ten KoRV proviral gene (DNA) positive koalas, including five with lymphoma, were also positive for the three KoRV viral 
gene (RNA) targets. Five koalas that were KoRV proviral gene (DNA) negative were also negative for the three KoRV viral gene (RNA) targets. Table 3 shows the comparative CT mean fold change $\left(2^{-\Delta \Delta C T}\right)$ and FC range $\left(2^{-(\Delta \Delta C T \pm s d)}\right)$ between KoRV positive and lymphomic koalas (expanded results Supplementary Material Table S1). Gene targets pol and env 1 had significantly higher expression for KoRV proviral and viral positive koalas with lymphoma versus those that were KoRV proviral and viral positive.

The targeted viral expression reiterated the expression demonstrated with RNAseq data, with the highest expression of KoRV genes in koalas with lymphoma. The koalas tested on both proviral (DNA) qPCR and viral (RNA) qPCR analyses demonstrated consistency in results, increasing confidence in these assays.

Table 3. Comparative CT method results (fold change $2^{-\Delta \Delta C T}$ ) of KoRV central genes from for lymph node RNA from KoRV positive koalas with lymphoma relative to KoRV positive koalas.

\begin{tabular}{cccc}
\hline \multirow{2}{*}{ KoRV Gene } & \multicolumn{2}{c}{$\mathbf{2}^{-\boldsymbol{\Delta \Delta C T}}$ Lymphomic Koalas Relative to Positive Koalas } \\
\cline { 2 - 4 } & Mean FC $^{*}$ & FC Range & $\boldsymbol{p}$ Value \\
\hline gag 2 & 299 & $47.7-1880.5$ & 0.060 \\
\hline pol & $\mathbf{6 0 2}$ & $185.8-1950.2$ & 0.012 \\
\hline env 1 & $\mathbf{2 4 2}$ & $42.5-1375.6$ & 0.012 \\
\hline
\end{tabular}

$\mathrm{FC}=$ Fold change calculated by $2^{-\Delta \Delta \mathrm{CT}} \cdot{ }^{*}$ Bold values are significant (Mann-Whitney $\mathrm{U}$ test, $\left.p<0.05\right)$, more detail of calculations and samples in Supplementary Material: Tables S1 and S2.

\section{Discussion}

The findings of this study demonstrate the complexity of KoRV status in SA koalas, but clearly show that the central region of KoRV ( $g a g$ 2, pol, env 1, and env 2) is diagnostic for classifying individuals as KoRV positive (infected) or negative (non-infected). The highest expression of all KoRV genes was by koalas with lymphoma, whereas non-lymphomic KoRV positive koalas had variable expression profiles in the central region of the KoRV viral genome. The koalas tested on both proviral (DNA) qPCR and viral (RNA) qPCR analyses demonstrated consistency in results. Gene target gag 1 was positive in almost all MLR koalas (215/216) regardless of KoRV status and the terminal sequences inclusive of this are present numerous times in the koala reference genome.

Based on the central region of KoRV, proviral PCR revealed two distinct groupings: $41.2 \%$ $(89 / 216)$ positive and $57.4 \%(124 / 216)$ negative for all targets. These results differ to those of a recent study in which MLR koalas were shown to have varying prevalence of each of the KoRV genes within their proviral DNA, with 79\% (77/97) PCR positive for at least one target on each proviral genes, 99\% (96/97) positive for pol gene target and only $41 \%$ (40/97) positive for one of the env gene targets [17]. Viral expression (qPCR) supported our proviral analyses since all KoRV negative koalas showed no expression of gag 2, pol, and env 1 targets. However, in this study there were a low number $(1.4 \%, 3 / 216)$ of koalas that showed mixed results in the proviral PCRs and this could be due to the sensitivity of these assays, as KoRV proviral load can be very low in SA koalas, or potentially represent acute infections.

Gene expression in KoRV positive koalas showed variable depth of coverage across the central region, except for lymphomic koalas in which it was consistently high. High expression of KoRV was shown directly from lymphomic tissue infected with exogenous KoRV and supports the ongoing assumption that oncogenesis occurs is associated with retroviral infection in koalas [22,23,34,35]. However, further studies to like causation beyond observation of upregulation of expression clonal transformed cells are needed and are the subject of future investigations with common KoRV insertion sites observed near oncogenes in koalas with cancer [36]. From RNAseq alignment to KoRV-A genome, it can be seen that there is more variance in the env region in the lymphoma samples than the KoRV positive samples (Figure 2), further investigations of the KoRV variants in this cohort could be undertaken to determine the role of different subtypes in oncogenesis, as found in other studies [5,37]. Suppression, latency, or subclinical infections of KoRV may explain the fragmentation of 
alignment to the central region of KoRV in KoRV positive, non-lymphomic koalas. In order for the host to survive, several anti-viral strategies have evolved in response to retroviral infections. Small piwi interacting RNA (piRNA) molecules or small interacting RNA (siRNA) have been found to silence transposons in germinal cells [38-40], DNA methylation can also inhibit transcription and several host genes have been shown to have antiretroviral properties [41]. piRNAs have been found in koalas, highly expressed within testicular tissue, but also expressed in other tissues including brain, liver, and lymph nodes [21]. The inhibition shown from piRNAs through ping-pong modelling in koalas, corresponds to central regions of the KoRV genome [21]. Therefore, piRNAs could be responsible for some of the inhibition and fragmentation of the transcription of central genes in SA koalas as seen in KoRV positive koalas. The low gene expression found in SA koalas differs to that found in previous studies of endogenised Queensland koalas, in which KoRV expression and viral loads are high $[17,23,25,42]$. Cellular genes that encode restriction factors, such as those belonging to the cytidine deaminases, have shown significant antiretroviral properties, including inhibition of viral transcription. Examples of antiretroviral cellular genes are APOBEC3, SAMHD1, and $M X 2$ [41]. A recent study found the addition of a mouse homolog of $A P O B E C 3$ significantly reduces the infectivity of KoRV in vitro [43]. The koala homolog to human or mouse APOBEC3 gene is not present, but $A P O B E C 1, A P O B E C 2$ APOBEC4, and AICDA genes (other cytidine deaminases) are annotated on the koala genome (NCBI database), with their effect yet to be demonstrated on KoRV infectivity.

From the RNAseq data, in two of the three KoRV proviral (DNA) negative koalas there was no detectable expression from the central region, but the other one koala showed a similar pattern of expression to the KoRV proviral (DNA) positive koalas. Unfortunately, this particular koala did not undergo viral (RNA) gene expression analysis through qPCR. The proviral PCRs were carried with blood or spleen and RNA-seq with lymph node tissue, this difference could support lymph nodes as the primary target for infection and replication. Therefore, our tentative conclusion is that this koala was recently infected with the virus only present in the lymph nodes and had not yet spread to other tissues including the blood and spleen. For those KoRV negative koalas without proviral DNA detection or proviral gene expression in the central region of the KoRV genome it would be highly unlikely that these koalas would be able to produce infectious KoRV virions. Env genes are responsible for entry into the cells, coding for the receptor binding domain and the transmembrane unit, while pol genes are responsible for enzymes including viral polymerase, gag genes code for structural proteins and allow for formation of a virion [44,45]. Therefore, without these elements, formation of KoRV virions would be impossible, but it would be necessary to carry out in vitro infection studies in cell culture to confirm this.

There were multiple sequences in the koala reference genome that showed strong homology to the KoRV terminal regions but not the central region. Some of these sequences have the KoRV central region replaced by sequences from the central region of $\mathrm{PhER}$. It is possible that these insertions in the reference genome have been generated by recombination between the termini of PhER and KoRV, attributed to recKoRV in previous studies [20,33]. We note that complete KoRV and PhER retroviral sequences only show $72 \%$ identity over 102 nucleotides around the 7500 bp region on the KoRV genome, which may be enough for recombination to have occurred. Additionally, the lack of KoRV in significant numbers of probable recKoRV positive koalas elicits evolutionary questions for this virus, unable to be answered without greater investigations. A possible alternative explanation for these terminal regions could be due to the historical foundation of this population, reintroduced to South Australia from very small numbers of Victorian koalas. Due to the genetic bottleneck, partial gene loss may have occurred in this population, with endogenisation of a defective version of KoRV in SA koalas. ERVs that have lost the env gene tend to have increased proliferation within the host genome, with less defined loci of integration [46], therefore the env-less terminal regions within this study could have increased proliferation, and in turn, increase transcription of KoRV fragments. Further 
long read genome sequencing of KoRV positive and negative koalas could help answer some of the questions surrounding these terminal regions.

There is the potential that the terminal retroviral expression in KoRV negative koalas could be inhibiting or reducing subsequent exogenous spread of KoRV in SA populations. These elements could also be reducing loads in KoRV positive koalas. Protective antiretroviral properties have been encountered in the study of endogenous retroviruses (ERVs). Some endogenous retroviral elements can inhibit other retroviral entry into cells and therefore increase the resistance to future retroviral infection, such as in feline leukemia virus (FeLV) and murine leukemia virus (MLV) [45,47-49]. Env regions have been shown to be recruited in response to exogenous retrovirus (XRV) infection to block viral receptors [50] and has been experimentally demonstrated in mice [49], cats [47], and chickens [51]. The transcribed KoRV fragments from the $5^{\prime}$ LTR and $5^{\prime}$ gag region and from the $3^{\prime}$ env and $3^{\prime}$ LTR could be part of the resistance to future KoRV infection, as suggested by Tarlinton et al. 2017 [19]. This would assume that these elements are endogenised throughout the population, which is likely since the RNA-seq alignment mapping showed presence of these regions in all koalas and proviral PCR analysis showed an overwhelming majority $(99.5 \%, 215 / 216)$ of koalas were positive for the $3^{\prime}$ gag gene target (gag 1$)$. In vitro studies could explore the ability for KoRV negative lymphocytes to withstand KoRV infection and the effect of transcripts from $5^{\prime}$ and $3^{\prime}$ regions of KoRV on infection rates. These studies would help distinguish clinical relevance of these KoRV transcripts and expand our understanding of KoRV transmission and cellular response.

\section{Conclusions}

South Australian koala populations are intriguing in their KoRV and other retroviral integration and expression patterns, differentiating them from their more extensively studied northern counterparts. The clinical relevance of KoRV in SA requires the definition between KoRV positive and KoRV negative koalas and this large cohort study demonstrated two clear groupings, allowing for future delineation. The commonly used pol qPCR should detect positive cases, however confirmation with gag 2 and env 1 gene targets would increase confidence, highlighting this central region as the most valuable for differentiation. This study also highlighted the homology to the terminal regions of KoRV in all koalas. Sequencing the termini and gap regions, or potentially long read genome sequencing, of KoRV negative SA koalas could increase understanding of what these regions are and how they are situated in the genome. In vitro cell culture studies using KoRV negative koalas could show whether they have a level of resistance to KoRV infection. KoRV negative koalas in SA are important for understanding not only infection and transmission, but also evolution of KoRV.

Supplementary Materials: The following are available online at https: / www.mdpi.com/article/ 10.3390 / ani11051477/s1, Table S1: Comparative CT method results for RNA from lymph nodes of koalas that were KoRV positive and KoRV positive diagnosed with lymphoma, using KoRV positive as the control group, Table S2: BLASTn analysis results of KoRV termini regions to the koala reference genome, Table S3: BLASTn analysis results of PhER to the koala reference genome.

Author Contributions: Conceptualization, F.H., N.S., T.S.; methodology, T.S., W.Y.L., F.H.; formal analysis, W.Y.L., R.T., T.S.; investigation, T.S.; resources, F.H., N.S., L.W., writing-original draft preparation, T.S.; writing - review and editing, T.S., N.S., R.T., F.H., W.Y.L., L.W.; supervision, F.H., N.S., L.W., R.T.; project administration, F.H., N.S.; funding acquisition, F.H., N.S., L.W. All authors have read and agreed to the published version of the manuscript.

Funding: This research was funded by The University of Adelaide HDR support fund grant number 13107182.

Institutional Review Board Statement: The sampling of koalas for this study was approved by the University of Adelaide Animal Ethics Committee, approval number: S-2016-169 and conducted in accordance with the guideline set out in the 'Australian Code for the care and use of animals for scientific purposes 8th edition (2013) (National Health and Medical Research Council: Canberra, 2013). 
Data Availability Statement: The data presented in this study are available on request from the corresponding author. The data are not publicly available due to ongoing research commitments.

Acknowledgments: We thank South Australian Koala Rescue groups, Adelaide Koala and Wildlife Hospital, Cleland Wildlife Park, and Adelaide veterinary clinics for their help with provision of koalas; Jessica Fabijan for her previous work and supply of samples from koalas with lymphoma; Kyneta Bhoola, Chloe Buchanen and Pathology rotation DVM students for their help in with necropsies; the Veterinary Diagnostics Laboratory, School of Animal and Veterinary Sciences, University of Adelaide for their support and help throughout, especially Adrian Hines and Rebecca Summerton; and A/P Rachael Tarlinton, University of Nottingham, for her comments on the manuscript.

Conflicts of Interest: The authors declare no conflict of interest.

\section{References}

1. Hanger, J.J.; Bromham, L.D.; McKee, J.J.; O’Brien, T.M.; Robinson, W.F. The nucleotide sequence of koala (Phascolarctos cinereus) retrovirus: A novel type C endogenous virus related to gibbon ape leukemia virus. J. Virol. 2000, 74, 4264-4272. [CrossRef]

2. Vogt, V.M. Retroviral Virions and Genomes. In Retroviruses; Coffin, J.M., Hughes, S.H., Varmus, H.E., Eds.; Cold Spring Harbor Laboratory Press: Cold Spring Harbor, NY, USA, 1997.

3. Shojima, T.; Hoshino, S.; Abe, M.; Yasuda, J.; Shogen, H.; Kobayashi, T.; Miyazawa, T. Construction and characterization of an infectious molecular clone of koala retrovirus. J. Virol. 2013, 87, 5081-5088. [CrossRef]

4. Shojima, T.; Yoshikawa, R.; Hoshino, S.; Shimode, S.; Nakagawa, S.; Ohata, T.; Nakaoka, R.; Miyazawa, T. Identification of a Novel Subgroup of Koala Retrovirus from Koalas in Japanese Zoos. J. Virol. 2013, 87, 9943-9948. [CrossRef] [PubMed]

5. Xu, W.; Stadler, C.K.; Gorman, K.; Jensen, N.; Kim, D.; Zheng, H.; Tang, S.; Switzer, W.M.; Pye, G.W.; Eiden, M.V. An exogenous retrovirus isolated from koalas with malignant neoplasias in a US zoo. Proc. Natl. Acad. Sci. USA 2013, 110, 11547-11552. [CrossRef] [PubMed]

6. Shimode, S.; Nakagawa, S.; Yoshikawa, R.; Shojima, T.; Miyazawa, T. Heterogeneity of koala retrovirus isolates. FEBS Lett. 2014, 588, 41-46. [CrossRef] [PubMed]

7. Xu, W.Q.; Gorman, K.; Santiago, J.C.; Kluska, K.; Eiden, M.V. Genetic diversity of koala retroviral envelopes. Viruses 2015, 7, 1258. [CrossRef]

8. Chappell, K.J.; Brealey, J.C.; Amarilla, A.A.; Watterson, D.; Hulse, L.; Palmieri, C.; Johnston, S.D.; Holmes, E.C.; Meers, J.; Young, P.R. Phylogenetic Diversity of Koala Retrovirus within a Wild Koala Population. J. Virol. 2017, 91. [CrossRef]

9. Simmons, G.; Young, P.; McKee, J.; Meers, J.; Mizuno, T. The epidemiology of Koala retrovirus. J. Vet. Epidemiol. 2011, 15, 1-9. [CrossRef]

10. Simmons, G.S.; Young, P.R.; Hanger, J.J.; Jones, K.; Clarke, D.; McKee, J.J.; Meers, J. Prevalence of koala retrovirus in geographically diverse populations in Australia. Aust. Vet. J. 2012, 90, 404-409. [CrossRef]

11. Stoye, J.P. Koala retrovirus: A genome invasion in real time. Genome Biol. 2006, 7, 241. [CrossRef] [PubMed]

12. Oliveira, N.M.; Farrell, K.B.; Eiden, M.V. In vitro characterization of a koala retrovirus. J. Virol. 2006, 80, 3104-3107. [CrossRef] [PubMed]

13. Fabijan, J.; Miller, D.; Olagoke, O.; Woolford, L.; Boardman, W.; Timms, P.; Polkinghorne, A.; Simmons, G.; Hemmatzadeh, F.; Trott, D.J.; et al. Prevalence and clinical significance of koala retrovirus in two South Australian koala (Phascolarctos cinereus) populations. J. Med. Microbiol. 2019, 68, 1072-1080. [CrossRef] [PubMed]

14. Fiebig, U.; Hartmann, M.G.; Bannert, N.; Kurth, R.; Denner, J. Transspecies transmission of the endogenous koala retrovirus. J. Virol. 2006, 80, 5651-5654. [CrossRef] [PubMed]

15. Legione, A.R.; Patterson, J.L.; Whiteley, P.; Firestone, S.M.; Curnick, M.; Bodley, K.; Lynch, M.; Gilkerson, J.R.; Sansom, F.M.; Devlin, J.M. Koala retrovirus genotyping analyses reveal a low prevalence of KoRV-A in Victorian koalas and an association with clinical disease. J. Med. Microbiol. 2017, 66, 236-244. [CrossRef]

16. Sarker, N.; Fabijan, J.; Seddon, J.; Tarlinton, R.; Owen, H.; Simmons, G.; Thia, J.; Blanchard, A.M.; Speight, N.; Kaler, J.; et al. Genetic diversity of Koala retrovirus env gene subtypes: Insights into northern and southern koala populations. J. Gen. Virol. 2019, 100, 1328-1339. [CrossRef] [PubMed]

17. Sarker, N.; Fabijan, J.; Owen, H.; Seddon, J.; Simmons, G.; Speight, N.; Kaler, J.; Woolford, L.; Emes, R.D.; Hemmatzadeh, F.; et al. Koala retrovirus viral load and disease burden in distinct northern and southern koala populations. Sci. Rep. 2020, 10, 263. [CrossRef] [PubMed]

18. Tarlinton, R.; Meers, J.; Young, P. Biology and evolution of the endogenous koala retrovirus. Cell. Mol. Life Sci. 2008, 65, 3413-3421. [CrossRef] [PubMed]

19. Tarlinton, R.E.; Sarker, N.; Fabijan, J.; Dottorini, T.; Woolford, L.; Meers, J.; Simmons, G.; Owen, H.; Seddon, J.M.; Hemmatzedah, F.; et al. Differential and defective expression of Koala Retrovirus reveal complexity of host and virus evolution. bioRxiv 2017. [CrossRef]

20. Lober, U.; Hobbs, M.; Dayaram, A.; Tsangaras, K.; Jones, K.; Alquezar-Planas, D.E.; Ishida, Y.; Meers, J.; Mayer, J.; Quedenau, C.; et al. Degradation and remobilization of endogenous retroviruses by recombination during the earliest stages of a germ-line invasion. Proc. Natl. Acad. Sci. USA 2018, 115, 8609-8614. [CrossRef] 
21. Yu, T.; Koppetsch, B.S.; Pagliarani, S.; Johnston, S.; Silverstein, N.J.; Luban, J.; Chappell, K.; Weng, Z.; Theurkauf, W.E. The piRNA Response to Retroviral Invasion of the Koala Genome. Cell 2019, 179, 632-643. [CrossRef]

22. Fabijan, J.; Woolford, L.; Lathe, S.; Simmons, G.; Hemmatzadeh, F.; Trott, D.J.; Speight, N. Lymphoma, Koala Retrovirus Infection and Reproductive Chlamydiosis in a Koala (Phascolarctos cinereus). J. Comp. Pathol. 2017, 157, 188-192. [CrossRef] [PubMed]

23. Tarlinton, R.; Meers, J.; Hanger, J.; Young, P. Real-time reverse transcriptase PCR for the endogenous koala retrovirus reveals an association between plasma viral load and neoplastic disease in koalas. J. Gen. Virol. 2005, 86, 783-787. [CrossRef] [PubMed]

24. Waugh, C.A.; Hanger, J.; Loader, J.; King, A.; Hobbs, M.; Johnson, R.; Timms, P. Infection with koala retrovirus subgroup B (KoRV-B), but not KoRV-A, is associated with chlamydial disease in free-ranging koalas (Phascolarctos cinereus). Sci. Rep. 2017, 7, 134. [CrossRef] [PubMed]

25. Fabijan, J.; Sarker, N.; Speight, N.; Owen, H.; Meers, J.; Simmons, G.; Seddon, J.; Emes, R.D.; Tarlinton, R.; Hemmatzadeh, F.; et al. Pathological Findings in Koala Retrovirus-positive Koalas (Phascolarctos cinereus) from Northern and Southern Australia. J. Comp. Pathol. 2020, 176, 50-66. [CrossRef]

26. Krueger, F. Trim Galore: A Wrapper Tool Around Cutadapt and FastQC to consistently Apply Quality And Adapter Trimming to FastQ Files. Available online: https://www.bioinformatics.babraham.ac.uk/projects/trim_galore/ (accessed on 8 October 2019).

27. Schubert, M.; Lindgreen, S.; Orlando, L. AdapterRemoval v2: Rapid adapter trimming, identification, and read merging. BMC Res. Notes 2016, 9, 88. [CrossRef]

28. Andrews, S. FastQC: A Quality Control Tool for High Throughput Sequence Data. Available online: http://www.bioinformatics babraham.ac.uk/projects/fastqc (accessed on 8 October 2019).

29. Kim, D.; Langmead, B.; Salzberg, S.L. HISAT: A fast spliced aligner with low memory requirements. Nat. Methods 2015, 12, 357-360. [CrossRef]

30. Altschul, S.F.; Gish, W.; Miller, W.; Myers, E.W.; Lipman, D.J. Basic local alignment search tool. J. Mol. Biol. 1990, 215 , 403-410. [CrossRef]

31. Coordinators, N.R. Database resources of the National Center for Biotechnology Information. Nucleic Acids Res. 2018, 46, D8-D13. [CrossRef]

32. Stanton, K.A.; Edger, P.P.; Puzey, J.R.; Kinser, T.; Cheng, P.; Vernon, D.M.; Forsthoefel, N.R.; Cooley, A.M. A Whole-Transcriptome Approach to Evaluating Reference Genes for Quantitative Gene Expression Studies: A Case Study in Mimulus. G3 Genes Genomes Genet. 2017, 7, 1085-1095. [CrossRef]

33. Hobbs, M.; King, A.; Salinas, R.; Chen, Z.; Tsangaras, K.; Greenwood, A.D.; Johnson, R.N.; Belov, K.; Wilkins, M.R.; Timms, P. Long-read genome sequence assembly provides insight into ongoing retroviral invasion of the koala germline. Sci. Rep. 2017, 7, 15838. [CrossRef]

34. Hanger, J. An Investigation of the Role of Retroviruses in Leukaemia and Related Diseases in Koalas; University of Queensland: Brisbane, Australia, 1999.

35. Kinney, M.E.; Pye, G.W. Koala Retrovirus: A Review. J. Zoo Wildl. Med. 2016, 47, 387-396. [CrossRef] [PubMed]

36. McEwen, G.K.; Alquezar-Planas, D.E.; Dayaram, A.; Gillett, A.; Tarlinton, R.; Mongan, N.; Chappell, K.J.; Henning, J.; Tan, M.; Timms, P.; et al. Retroviral integrations contribute to elevated host cancer rates during germline invasion. Nat. Commun. 2021, 12, 1316. [CrossRef] [PubMed]

37. Quigley, B.L.; Ong, V.A.; Hanger, J.; Timms, P.; Simon, V. Molecular Dynamics and Mode of Transmission of Koala Retrovirus as It Invades and Spreads through a Wild Queensland Koala Population. J. Virol. 2018, 92. [CrossRef]

38. Ahlenstiel, C.; Mendez, C.; Lim, S.T.; Marks, K.; Turville, S.; Cooper, D.A.; Kelleher, A.D.; Suzuki, K. Novel RNA Duplex Locks HIV-1 in a Latent State via Chromatin-mediated Transcriptional Silencing. Mol. Ther. Nucleic Acids 2015, 4, e261. [CrossRef] [PubMed]

39. Fu, Q.; Wang, P.J. Mammalian piRNAs: Biogenesis, function, and mysteries. Spermatogenesis 2014, 4, e27889. [CrossRef]

40. Tarazona, S.; Garcia-Alcalde, F.; Dopazo, J.; Ferrer, A.; Conesa, A. Differential expression in RNA-seq: A matter of depth. Genome Res. 2011, 21, 2213-2223. [CrossRef]

41. Goff, S.P. Cellular Factors That Regulate Retrovirus Uncoating and Reverse Transcription. In Retrovirus-Cell Interactions; Parent, L.J., Ed.; Academic Press: London, UK, 2018; pp. 51-112.

42. Olagoke, O.; Miller, D.; Hemmatzadeh, F.; Stephenson, T.; Fabijan, J.; Hutt, P.; Finch, S.; Speight, N.; Timms, P. Induction of neutralizing antibody response against koala retrovirus (KoRV) and reduction in viral load in koalas following vaccination with recombinant KoRV envelope protein. NPJ Vaccines 2018, 3, 30. [CrossRef]

43. Nitta, T.; Ha, D.; Galvez, F.; Miyazawa, T.; Fan, H. Human and murine APOBEC3s restrict replication of koala retrovirus by different mechanisms. Retrovirology 2015, 12, 68. [CrossRef]

44. Balasuriya, U.B.R.; Barratt-Boyes, S.; Beer, M.; Bird, B.; Brownlie, J.; Coffey, L.L.; Cullen, J.M.; Delhon, G.A.; Donis, R.O.; Gardner, I.; et al. Retroviridae. In Fenner's Veterinary Virology, 5th ed.; MacLachlan, N.J., Dubovi, E.J., Eds.; Academic Press: London, UK, 2017; pp. 269-297.

45. Greenwood, A.D.; Ishida, Y.; O’Brien, S.P.; Roca, A.L.; Eiden, M.V. Transmission, Evolution, and Endogenization: Lessons Learned from Recent Retroviral Invasions. Microbiol. Mol. Biol. Rev. 2018, 82. [CrossRef] [PubMed]

46. Magiorkinis, G.; Gifford, R.J.; Katzourakis, A.; De Ranter, J.; Belshaw, R. Env-less endogenous retroviruses are genomic superspreaders. Proc. Natl. Acad. Sci. USA 2012, 109, 7385-7390. [CrossRef] 
47. Mcdougall, A.S.; Terry, A.; Tzavaras, T.; Cheney, C.; Rojko, J.; Neil, J.C. Defective Endogenous Proviruses Are Expressed in Feline Lymphoid Cells: Evidence for a Role in Natural Resistance to Subgroup B Feline Leukemia Viruses. J. Virol. 1994, 68, 2151-2160. [CrossRef] [PubMed]

48. Nethe, M.; Berkhout, B.; van der Kuyl, A.C. Retroviral superinfection resistance. Retrovirology 2005, 2, 52. [CrossRef] [PubMed]

49. Wu, T.; Yan, Y.; Kozak, C.A. Rmcf2, a xenotropic provirus in the Asian mouse species Mus castaneus, blocks infection by polytropic mouse gammaretroviruses. J. Virol. 2005, 79, 9677-9684. [CrossRef] [PubMed]

50. Stoye, J.P. Studies of endogenous retroviruses reveal a continuing evolutionary saga. Nat. Rev. Microbiol. 2012, 10, 395-406. [CrossRef]

51. Robinson, H.L.; Lamoreux, W.F. Expression of Endogenous ALV Antigens and Susceptibility to Subgroup E ALV in Three Strains of Chickens (Endogenous Avian C-Type Virus). Virology 1976, 69, 50-62. [CrossRef] 